\title{
ПОНЯТИЙНЫЙ БАЗИС И ЕГО РОЛЬ В ФОРМИРОВАНИИ НОВЫХ РАЗДЕЛОВ МАТЕМАТИКИ
}

\section{THE CONCEPTUAL BASIS AND ITS ROLE IN THE FORMATION OF NEW SECTIONS OF MATHEMATICS}

\section{Poberezhnyy}

Summary: The article considers the state of the conceptual basis of mathematics in the historical and philosophical aspect. The genesis and evolution of some of the most ambiguous mathematical concepts are shown. The article describes how the evolution of concepts leads to the development of new scientific theories. On the other hand, the emergence of new directions in mathematics contributes to the renewal of its conceptual apparatus.

Keywords: history of mathematics, conceptual basis, irrational numbers, infinitesimal quantities, continuum, transfinite numbers.

\author{
Побережный Иван Александрович \\ Аспирант, Курский государственный университет \\ ivan.poberezhnyy@gmail.com
}

Аннотация: В статье рассмотрено состояние понятийного базиса математики в историко-философском аспекте. Показаны генезис и эволюция некоторых наиболее неоднозначных математических понятий. Описано, как эволюция понятий приводит к разработке новых научных теорий. С другой стороны, возникновение новых направлений в математике способствует обновлению ее понятийного аппарата.

Ключевые слова: история математики, понятийный базис, иррациональные числа, бесконечно малые величины, континуум, трансфинитные числа.

нальных чисел приписывают пифагорейцу Гиппасу из Метапонта [10, с.56-57]. Согласно учению пифагорейской школы, существует достаточно маленькая, неделимая единица длины, которая могла бы равномерно вписаться в любую из длин. Гиппасу ещё в $\mathrm{V}$ веке до нашей эры удалось доказать, что такой единой единицы измерения не существует и что утверждение о таком существовании на самом деле приводит к противоречию. Он сделал это, показав, что если бы гипотенуза равнобедренного прямоугольного треугольника была соизмерима с катетом, то одна из этих длин должна была бы быть одновременно нечетной и четной, что невозможно. Греческие математики назвали это отношение несоизмеримых величин а入оүос (невыразимое). Открытие несоизмеримых отрезков прямой указывало на новую проблему, ставшую перед греками: отношение дискретного к непрерывному [3].

Евдокс Книдский разработал новое понимание теории пропорций, которое учитывало не только соизмеримые, но и несоизмеримые величины. Величина " ... не была числом, а обозначала такие объекты, как отрезки, углы, площади, объемы и время, которые могли изменяться, как мы сказали бы, непрерывно. Величины противопоставлялись числам, которые перескакивали от одного значения к другому, как от 4 до 5» [13, p.48]. Разработка Евдоксом теории пропорций создала прочную базу для понимания и использования иррациональных чисел в математике.

В Средние века развитие математики наиболее су-

Первое доказательство существования иррацио- 
щественно проявилось в странах Востока. Создание арабскими математиками алгебры приводит к тому, иррациональные числа рассматриваются как алгебраические объекты. В X веке арабский математик Аль-Хашими представил общие рассуждения и доказательства для иррациональных чисел, где он рассматривал умножение, деление и другие арифметические операции. Многие из этих концепций позже были приняты европейскими математиками.

Дальнейшим шагом в развитии теории иррациональных чисел стало решение вопроса о том, все ли числа являются алгебраическими, т. е. корнями алгебраических многочленов с целыми коэффициентами. В 1844 году французский математик Ж. Лиувилль впервые привел пример трансцендентного (т. е. неалгебраического) числа [14]. Множества алгебраических и трансцендентных чисел существенным образом отличаются друг от друга. Множество алгебраических чисел бесконечно и счётно, т.е. эквивалентно множеству натуральных чисел, замкнуто относительно операций сложения, вычитания, умножения и деления (исключая деление на 0). Множество трансцендентных чисел несчётно. Таким образом, трансцендентных чисел «больше», чем алгебраических. Их слишком много, чтобы можно было представить в виде последовательности.

Понятие непрерывности в математике имеет давнюю историю. Значение слова непрерывный - «сущность, которая не имеет промежутков». Обычно считается, что пространство и время непрерывны, и некоторые философы утверждали, что все естественные процессы происходят непрерывно. Например, Г. Лейбниц утверждал, что «природа не совершает скачка» (natura non facit saltus) [11]. Быть непрерывным - значит образовывать нечто неразрывное, сплошное. Непрерывная сущность - континуум - не имеет пробелов. Сущность, противоположная непрерывности, - дискретность. Дискретное состоит из отдельных точек, элементов, жестко разделенных между собой. Непрерывное подобно небу или океану, дискретное подобно гальке на пляже или листьям на дереве. Непрерывность означает единство; дискретность - множественность.

В математике понятию непрерывности на протяжении истории давали всё более и более точные определения. Так, в конце XVIII века непрерывность функции означала, что бесконечно малые изменения значения аргумента индуцируют бесконечно малые изменения значения функции. В XIX веке это определение связывают с понятием предела.

Традиционно бесконечно малая величина - это последовательность, переменная величина, такая, которая меньше любой конечной величины. Практически величина является бесконечно малой, если ее квадратом и всеми более высокими степенями можно пренебречь. В теории пределов бесконечно малой является последовательность, предел которой равен нулю. Таким образом, непрерывные кривые считались составленными из бесконечно малых прямых линий.

Бесконечно малые встречаются в математике греческого философа-атомиста Демокрита (V в. до н.э.), но уже отсутствуют в математике Евклида. Приняв не вполне понятную форму «неделимых», они вновь появляются в европейской математике в эпоху позднего средневековья. B XIX веке с появлением и развитием теории пределов математики на какое-то время отказываются от понятия бесконечно малых в связи с отсутствием его четкой определенности [1. с.230]. Однако позже понятие бесконечно малого было переосмыслено на более строгой основе.

Фундаментальная природа континуума состоит в неделимости, при этом любой континуум допускает бесконечное последовательное деление. В древности это утверждение встречало возражение. Основатель школы атомизма Демокрит утверждал, что существуют атомы, неделимые частицы, что для всего существует предел деления, и что не только материя, но и сама протяженность не является бесконечно делимой. В то же самое время атомисты утверждали, что непрерывное в конечном счете сводимо к дискретному, как на уровне ощущений, так и на уровне философствования. В XIX веке учение Демокрита получает триумфальное признание в физике и химии, и понятие континуума смещается в область математического познания.

Бесконечно малая величина была понята как «конечная часть континуума». Если утверждается, что дискретная сущность состоит из отдельных единиц, можно аналогичным образом утверждать, что континуум составлен из бесконечно малых величин, как своих частей. Но каждая часть континуума делима, так как сама является континуумом. Это значит, что точка, как неделимый объект, не может быть частью континуума. Иными словами, бесконечно малые величины, как части континуумов, не могут быть точками.

Конечные величины принимаются как нечто экстенсивное, локализованное, как масса или объем, которые определяются в протяженных областях пространства. Напротив, бесконечно малые величины были истолкованы как интенсивные величины, локально определенные, такие как температура или плотность. Результатом «распределения» или «интегрирования» интенсивной величины по такой интенсивной величине является превращение первой в бесконечно малую экстенсивную величину: таким образом, температура преобразуется в бесконечно малую энергию, «теплоту», а плотность - в бесконечно малую массу. Когда континуум определяется 
движением, связанные с ним бесконечно малые интенсивные величины идентифицируются как потенциальные величины.

Бесконечно малое число - это число, которое, хотя и не совпадает с нулем, в некотором смысле меньше любого конечного числа. Неделимое - это то, что обычно понимается как не состоящее из частей.

Понятие бесконечно малого всегда подвергалось жесткой критике, каким бы полезным оно ни было на практике. В XVIII веке Дж. Беркли называл бесконечно малые «призраками ушедших величин» [15], в XIX веке Г. Кантор сравнивал их с «бациллами холеры», заражающими математику, а в XX веке Б. Рассел резко критиковал как «ненужные, ошибочные и самопротиворечивые», как «логически сомнительные сущности» [7]. Тем не менее бесконечно малые величины широко использовались в различных дисциплинах преимущественно практической направленности, к примеру, в дифференциальной геометрии, физике, технических науках.

В шестидесятых годах прошлого века американский математик А. Робинсон, используя методы математической логики, создал нестандартный анализ, расширение математического анализа, охватывающее бесконечно большие и бесконечно малые величины, развивая идею, которая, по существу, восходит к Лейбницу. И наконец, в семидесятые годы прошлого столетия разрабатывается гладкий инфинитезимальный анализ, основанный на идеях У. Ловера и использующий методы теории категорий, он рассматривает все функции как непрерывные и невыражаемые через дискретные элементы. Данная теория может быть интерпретирована как описывающая мир, в котором линии состоят из бесконечно малых отрезков, а не из точек. Развитие данных теорий вдохнуло новую жизнь в понятие бесконечно малого и дало новое понимание природы континуума.

Противопоставление непрерывности и дискретности, имевшее место в древнегреческой философии, вытекает из более фундаментального вопроса о едином и множественном, лежащего в основе ранней греческой философии. Элеатские философы VI-V вВ. до н.э. Парменид и Зенон, утверждали, что понимание бытия как делимого на части ведет к противоречию, что мир представляет собой статичное, неизменное единство [5, с.235]. Парменидово бытие - это континуум без частей, одновременно континуум и атом. Учение об атомизме, возникшее как попытка избежать Элеатской дилеммы, было прежде всего физической теорией. Левкипп и Демокрит утверждали, что материя не бесконечно делима, а состоит из неделимых, твердых, однородных, пространственно протяженных частиц.

Атомизм был оспорен Аристотелем, который первым предпринял систематический анализ непрерывности и дискретности. Он утверждал, что физическая реальность - это непрерывная полнота и что структура континуума, общая пространству, времени и движению, не может быть сведена ни к чему иному. По мнению Аристотеля, непрерывные величины потенциально делимы до бесконечности.

Аристотель определяет непрерывность и дискретность как атрибуты, относящиеся к категории количества. Такие величины, как прямые и плоскости, пространство и время непрерывны в силу того, что их составные части соединяются вместе на некоторой общей границе. В то же время никакие составные части дискретной величины не могут иметь общей границы. Один из центральных тезисов Аристотеля - несводимость континуума к дискретному, обосновывается тем, что континуум не может быть «составлен» из неделимых частей. Аристотель утверждал, что непрерывная величина не может состоять из точек, единиц, лишенных протяженности [9].

Философы-стоики (IIIв. до н.э.) Зенон и Хрисипп отстаивали аристотелевское положение о том, что пространство, время, материя и движение непрерывны. Все физические явления они рассматривали как связанные посредством растягивающих сил в пневме, а сама материя считалась производной от связывающих свойств пневмы, которую она содержит [2].

Философы средневековой Европы, подчиняясь огромному авторитету Аристотеля, в основном в той или иной форме придерживавшиеся его позиции. Хотя некоторые ученые того времени, например Г. Харклей, отстаивали идеи атомизма и попытались согласовать их с учением Аристотеля. Их идеи встретили опровержение со стороны Иоанна Дунса Скота, в котором он предлагает чисто геометрические аргументы против композиции континуума из неделимых элементов. Один из этих аргументов состоит в том, что если бы диагональ и сторона квадрата состояли из точек, то они были бы соизмеримы. У. Оккам признает, что на произвольно малых отрезках прямой должно лежать бесконечно много точек, но из этого не следует, что линии или вообще любой континуум состоят из точек.

Интересны взгляды на континуум Николая Кузанского, который утверждал, что любой континуум делим в двух смыслах: идеальном и реальном. Идеальное деление прогрессирует до бесконечности; действительное деление завершается в атомах через конечное число шагов. Концепция реального бесконечного у Н. Кузанского отражена в его квадратуре круга.

Исчисление бесконечно малых величин, сформировавшееся в XVI-XVII веках и имевшее своим основным предметом непрерывную вариацию, можно рассматри- 
вать как своего рода синтез непрерывного и дискретного, причем бесконечно малые величины заполняют промежуток между ними. Таким образом, именно бесконечно малое должно было служить математической ступенью между непрерывным и дискретным.

Г. Галилей считал, что бесконечность неделимых никогда не будет получена последовательным делением, но он находит метафизическое применение идеи рассмотрения круга как бесконечно-стороннего многоугольника. Когда прямая изгибается в окружность, Галилей утверждает, что линия может была разделена на неделимые части, точки. Если эти части являются сторонами бесконечно-стороннего многоугольника, то их можно характеризовать не как неделимые точки, а как несгибаемые прямые, каждая из которых одновременно является частью окружности и касательной к окружности [4].

Р. Декарт использовал в своих математических работах методы бесконечно малых. Декарт различает бытие протяженное (res extensa) и бытие мыслящее (res cogitans) на том основании, что первое, будучи пространственнопротяженным, делимо, тогда как второе, ментальное, не имеет частей. Следствием отождествления материи и пространственной протяженности является то, что материя непрерывна и бесконечно делима. Таким образом, пространство у Декарта, как и у стоиков, - это объем, заполненный непрерывной средой [5, с.301].

В 1873 году Кантор показал, что рациональные числа, хотя и бесконечны, являются счетными (или исчисляемыми), потому что они могут быть поставлены в однозначное соответствие с натуральными. Он показал, что множество действительных чисел бесконечно и неисчислимо, и что трансцендентные числа, которые являются подмножеством иррациональных, неисчислимы и поэтому более многочисленны, чем целые числа, которые должны быть поняты как бесконечные [12, р.262]. Развивая новые способы постановки вопросов о непрерывности и бесконечности, Кантор вызвал споры в научном мире тем, что он объединил свою теорию с платоновской метафизикой. В то же время Кронекер, считавший, что существуют только целые числа («Бог создал целые числа, а все остальное - дело рук человека»), в течение многих лет настойчиво отвергал его рассуждения [7, с. 85].

В 1883 году Г. Кантор вводит понятие ординала или порядкового числа. Новое множество представляет собой расширение множества натуральных чисел. Над ординалами можно производить те же арифметические действия, что и над другими числами - сложение, умножение, возведение в степень. Трансфинитные числа - это бесконечные порядковые числа. Данные числа сыграли важную роль в доказательстве различных теорем теории множеств. Особенно ценным оказался в данном случае принцип трансфинитной индукции.
В математике трансфинитные числа - это числа, которые бесконечны в том смысле, что они больше всех конечных чисел, но не обязательно абсолютно бесконечны. Эти числа можно разделить на два типа: трансфинитные кардиналы и трансфинитные ординалы. Трансфинитные кардиналы - это бесконечные числа, используемые для количественной оценки различных размеров бесконечности, которых существует множество. Помимо трансфинитных кардиналов, существуют также трансфинитные ординалы. Это числа, используемые для описания порядка множеств.

В 1895-97 годах Кантор изложил свой взгляд на непрерывность и бесконечность, включая бесконечные ординалы и кардиналы, в своей работе «К обоснованию учения о трансфинитных множествах» [6, с. 173-245]. Эта работа содержит его концепцию трансфинитных чисел, к которой он пришел, продемонстрировав, что бесконечное множество может быть помещено в взаимно однозначное соответствие с одним из его подмножеств.

Трансфинитные числа, которые были введены Кантором, известны в обозначении, которое он принял для них позже: в виде буквы к (алеф) 一 первой буквы еврейского алфавита. Этой буквой обозначается мощность, то есть число элементов бесконечного множества, так что отношения эквивалентности между бесконечными множествами часто выражают через трансфинитные кардинальные числа, алефы.

Под наименьшим трансфинитным кардинальным числом он имел в виду кардинальное число любого множества, которое может быть помещено в однозначное соответствие с целыми положительными числами. Это трансфинитное число он называл алеф-нуль. Большие трансфинитные кардинальные числа обозначались алефодин, алеф-два, ... Затем он разработал арифметику трансфинитных чисел, аналогичную конечной арифметике.

Г. Кантор показал, что множество точек на прямой обладает большим кардинальным числом, чем алеф-нуль. Это привело к проблеме гипотезы континуума, состоявшей в том, что нет кардинальных чисел между алеф-нуль и кардинальным числом точек на прямой. Эта проблема представляла большой интерес для математического мира и изучалась многими последующими математиками, включая К. Геделя и П. Коэна.

На рубеже веков работы были окончательно признаны фундаментальными для развития теории функций, анализа и топологии. Более того, его работа стимулировала в дальнейшем развитие как интуитивистской, так и формалистской школ в логических основах математики. Благодаря Г. Кантору понятие актуальной бесконечности стало доступно для строгого, формально-логического и математического анализа. 
Однако теория множеств Г. Кантора была воспринята в научном мире неоднозначно. Так, в то время как Д. Гильберт считал теорию Кантора «высочайшим проявлением математического гения», убежденными противниками этой теории были Л. Кроннекер и А. Пуанкаре. Пуанкаре даже назвал теорию трансфинитных чисел «болезнью», от которой, как он надеялся, математика когда-нибудь должна будет излечиться [8, с.42].
Таким образом, понятийный базис математики, как и понятийный аппарат любой другой науки, играет большую роль в ее развитии. Эволюция понятий приводит к разработке новых научных теорий и новых разделов математики, что неоднократно имело место на протяжении истории математики. С другой стороны, возникновение новых направлений в математике обновляло ее понятийный аппарат, уточняло понятийный базис.

\section{ЛИТЕРАТУРА}

1. Арепьев Е.И. Природа чисел в свете расширенной трактовки действительности // Российский гуманитарный журнал. 2014. Т. 3. № 4. С. $229-236$.

2. Ван дер Варден Б.Л. Пробуждающая наука. Математика др. Египта, Вавилона и Греции - [Пифагорейское учение о гармонии]. Пер. с голланд. и замеч. И.В. Веселовского. - М.: Физматгиз, 1959. - 459 с.

3. Гайденко П.П. Греческая философия в ее связи с наукой / П.П. Гайденко. - М., 2000. - 319 с.

4. Галилей Г. Пробирных дел мастер. - М., 1987. - 272 с.

5. Даан-Дальмедико А., Пейффер Ж. Пути и лабиринты. Очерки по истории математики/Пер. с франц. А.А. Брядинской. Под ред. И.Г. Башмаковой. М.: Мир, 1986. -431 c.

6. Кантор Г. Труды по теории множеств. М.: Наука, 1985. - 431 с.

7. Клайн М. Математика. Утрата определенности: пер. с англ. / под ред., с предисл. и примеч. И.М. Яглома. - М.: Мир, 1984. - 434 с.

8. Михайлова Н.В. Теория бесконечных множеств Кантора в контексте генезиса философии математики / Математические структуры и моделирование. 2015. №4(36). C. 40-48

9. Никаноров С.Н. Число у Аристотеля и в философии Нового времени. URL: http://turba-philosophorum.narod.ru /forskninger/Nikanorov/1_Num_Arist.html (Дата обращения 10.06.2021)

10. Чанышев А.Н. Италийская философия. М.: Издательство Московского государственного университета, 1975. - 216 с.

11. Continuity and Infinitesimals/ https://plato.stanford.edu/entries/continuity/

12. Godel, K. What is Cantor's continuum problem / K. Gogel // Philosophy of Mathematics: Selected Readings. - N.Y.: Englewood Hill, 1964. - P. 258-273.

13. Kline, M. (1990). Mathematical Thought from Ancient to Modern Times, Vol. 1. New York: Oxford University Press (original work published 1972)

14. Hyun Seok, Lee. On Transcendence Theory with little history, new results and open problems. URL: https://www.math.sci.hokudai.ac.jp/ wakate /mcyr/2016/pdf/ LEE.pdf (Дата обращения 10.06.2021)

15. The Analyst; or, a Discourse Addressed to an Infidel Mathematician (1734) by George Berkeley (1685-1753). URL: https://en.wikisource.org/wiki/The_Analyst:a_ Discourse_addressed_to_an_Infidel_Mathematician\#XVI (Дата обращения 10.06.2021)

○ Побережный Иван Александрович (ivan.poberezhnyy@gmail.com).

Журнал «Современная наука: актуальные проблемы теории и практики» 